\title{
Selección de los esquemas y sitios de asociación para mezclas complejas
}

\section{Selection of association schemes and sites for complex mixtures}

\author{
págs. 84-93 \\ Grupo de investigación: Procesos de Separación no Convencionales \\ Línea de investigación: Proceso de separación sin reacción \\ Yovanny Morales Hernández• Jaime Arturo Calvache•• María Monguí •.• Cristian Rodríguez ...
}

Recibido: agosto 01 de 2016 Aceptado: noviembre 25 de 2016

\begin{abstract}
RESUMEN
Los modelos y ecuaciones de estado convencionales, no pueden predecir satisfactoriamente el equilibrio de fases en mezclas asociativas, por lo cual, se desarrollaron modelos termodinámicos basados en la teoría de la perturbación de Wertheim que involucran la relación entre la energía residual de Helmholtz debido a la asociación y la fracción de moléculas no asociadas en un sitio, describiendo así el fenómeno de asociación. En este trabajo, se muestra la selección de los esquemas y sitios de asociación, como también las ecuaciones de la propiedad clave en el término de asociación planteadas a partir de la formación de puentes de hidrógeno entre sistemas binarios de ácido + ácido, ácido + agua, alcohol + alcohol y alcohol + agua.
\end{abstract}

Palabras clave: equilibrio de fases, puente de hidrógeno, ácido carboxílico, alcohol.

\begin{abstract}
The classical thermodynamic models cannot predict satisfactory the phase equilibria on associative mixtures, thermodynamic models based on Wertheim's perturbation theory were developed to overcome this drawback; such models involved the relation between the residual Helmholtz energy due to the association and fraction of molecules not bonded on a site, that describes the association phenomena. This paper shows the selection of schemes and association sites, and also equations of the key property in association terms, made from the hydrogen bonding formation between binary systems of acid + acid, acid + water, alcohol + alcohol and alcohol + water.
\end{abstract}

Key words: phase equilibria, hydrogen bonding, carboxylic acid, alcohol

\footnotetext{
- Docente investigador, Ingeniero Químico. Fundación Universidad de América. yovanny.morales@profesores.uamerica.edu.co -• Docente investigador, Ingeniero Químico, M. Sc. Fundación Universidad de América. jaime.arturo@profesores.uamerica.edu.co .. Co investigador, Ingeniera Química. Fundación Universidad de América maria.mongui@estudiantes.uamerica.edu.co

... Co investigador, Ingeniero Químico, Fundación Universidad de América cristian.rodriguez2@estudiantes.uamerica.edu.co
} 


\section{INTRODUCCIÓN}

Los modelos termodinámicos para mezclas que contienen componentes con la capacidad de formar puentes de hidrógeno, incluyen la contribución de la teoría química responsable de la formación de productos de asociación. Modelos como SAFT (Chapman et al. 1989 \& Chapman et al. 1990) y CPA (Kontogeorgis et al. 1996) fueron desarrollados para describir el fenómeno de asociación y predecir el equilibrio de fases en mezclas que contengan componentes como agua, ácidos carboxílicos, alcoholes, aminas, fenoles, glicoles y otras sustancias capaces de formar puentes hidrógeno.

El objetivo de este artículo es exponer el planteamiento de las ecuaciones empleadas en el cálculo de la relación clave del término de asociación $X_{A}$, utilizado en los modelos termodinámicos SAFT y CPA.

\section{MÉTODOS}

\section{Término de asociación}

El término de asociación representa la contribución en la energía residual de Helmholtz (Michelsen \& Hendriks, 2001) cuando se presentan interacciones de puentes de hidrógeno en un sistema.

El término de asociación para mezclas está dado por la ecuación:

$$
\frac{a^{a s s o c}}{R T}=\sum_{i} x_{i} \sum_{A_{i}}\left(\ln X_{A_{i}}-\frac{X_{A_{i}}}{2}+\frac{1}{2}\right)
$$

Donde $X_{A_{j}}$ es la fracción de moléculas i no asociadas al sitio A y puede ser determinada por:

$$
X_{A_{l}}=\frac{1}{1+\rho \sum_{j} x_{j} \sum_{B_{j}} X_{B_{j}} \Delta^{A_{i} B_{j}}}
$$

Siendo la densidad molar y $\Delta^{A_{i} B_{i}}$ la fuerza de asociación entre dos sitios $A$ y $B$ que pertenecen a dos moléculas diferentes i y j.

La fuerza de asociación para la ecuación SAFT está dada por:

$$
\Delta^{A_{i} B_{j}}=d_{i j}^{3} g_{i j}\left(d_{i j}\right)^{\text {seg }} \boldsymbol{\kappa}^{A_{i} B_{j}}\left[\exp \left(\boldsymbol{\varepsilon}^{A_{i} B_{j}} / \boldsymbol{\kappa} T\right)-1\right]
$$

Y para la ecuación de estado CPA esta expresada como:

$$
\Delta^{A_{i} B_{j}}=d_{i j}^{3} g_{i j}\left(d_{i j}\right)^{\text {seg }} \boldsymbol{\kappa}^{A_{i} B_{j}}\left[\exp \left(\frac{\varepsilon^{A_{i} B_{j}}}{R T}\right)-1\right] b_{i j} \beta^{A_{i} B_{j}}
$$




\section{Aplicación para componente puro}

Para estimar propiedades de componente puro, como la presión de vapor, densidades y propiedades críticas, es importante seleccionar un esquema adecuado que represente la asociación entre moléculas, para ello, Huang y Radosz (1990) proponen una clasificación simplificada de moléculas de acuerdo al número y tipos de sitios donde ocurre la formación de puentes de hidrógeno, como se presenta en la tabla 1.

Además, Huang y Radosz (1990) establecen como punto de partida una aproximación en el cálculo de la fracción $X_{A}$ que depende del tipo de sitio de asociación seleccionado, como se muestra en la tabla 2.

\section{Aplicación para mezclas}

Para la predicción del equilibrio de fases de mezclas asociativas no es posible utilizar una solución analítica en el cálculo de las fracciones $X_{A_{A}}$, por lo tanto es necesario emplear una solución numérica para resolver el sistema de ecuaciones génerado por la interacción entre moléculas según la cantidad de sitios seleccionados (Michelsen, 2006). En la tabla 3 se presentan mezclas binarias con sus respectivos esquemas de asociación usados en este trabajo.

\section{RESULTADOS Y DISCUSIÓN}

\section{Mezclas de ácidos}

Las mezclas de ácidos carboxílicos son representados usando el modelo de asociación $1 \mathrm{~A}$, que consiste en un único sitio que actúa como aceptor-donador de protones, así como se muestra en la figura 1.

Las interacciones entre moléculas de un ácido i y un ácido j se dan por la formación de puentes de hidrógeno entre sitios.

- Sitio $A_{1}$ de una molécula i + sitio $A_{1}$ de otra molécula i

- Sitio $A_{1}$ de una molécula i + sitio $A_{2}$ de una molécula j

- Sitio $A_{2}$ de una molécula j + sitio $A_{2}$ de otra molécula j

A partir de lo anterior, la ecuación 2 para mezclas de ácidos se expresa como:

$$
\begin{aligned}
& X_{A_{1}}=\frac{1}{1+\rho\left(x_{1} X_{A_{1}} \Delta^{A_{1} A_{1}}+x_{2} X_{A_{2}} \Delta^{A_{1} A_{2}}\right)} \\
& X_{A_{2}}=\frac{1}{1+\rho\left(x_{1} X_{A_{1}} \Delta^{A_{1} A_{2}}+x_{2} X_{A_{2}} \Delta^{A_{2} A_{2}}\right)}
\end{aligned}
$$




\section{Mezclas de ácidos y agua}

Las mezclas de ácidos carboxílicos y agua, son representadas con los esquemas de asociación $1 \mathrm{~A}$ y $4 \mathrm{C}$, respectivamente. Sin embargo, algunos investigadores emplean el modelo de asociación 3B, pero el esquema 4C presenta los mejores resultados (Folas, 2006). La molécula de agua está conformada por 2 sitios aceptores y 2 sitios donadores de protones, como se presenta en la figura 2 . Los sitios donadores de protones están conformados por átomos de hidrógeno y tienen la capacidad de formar puentes de hidrógeno con electrones no enlazados de una molécula (aceptores de protones).

De acuerdo con lo anterior, las interacciones entre moléculas de ácido (i) y agua (j) para la formación de puentes de hidrógeno está dada por:

Sitio $A_{1}$ de una molécula i + sitio $A_{1}$ de otra molécula i

Sitio $A_{1}$ de una molécula i + sitio $A_{2}$ de una molécula j

Sitio $A_{2}$ de una molécula j + sitio $B_{2}$ de otra molécula j

Sitio $A_{1}$ de una molécula i + sitio $C_{2}$ de una molécula j

Sitio $A_{1}$ de una molécula $\mathrm{i}+$ sitio $D_{2}$ de una molécula j

Sitio $A_{2}$ de una molécula j + sitio $C_{2}$ de otra molécula j

Sitio $A_{2}$ de una molécula j + sitio $D_{2}$ de otra molécula j

Sitio $B_{2}$ de una molécula $\mathrm{j}+$ sitio $C_{2}$ de otra molécula $\mathrm{j}$

Sitio $B_{2}$ de una molécula j + sitio $D_{2}$ de otra molécula j

Teniendo en cuenta la formación de enlaces, las ecuaciones para el cálculo de la fracción $X_{\bar{A}_{i}}$ se expresan como:

$$
\begin{gathered}
X_{A_{1}}=\frac{1}{1+\rho\left(x_{1} X_{A_{1}} \Delta^{A_{1} A_{1}}+x_{2} X_{A_{2}} \Delta^{A_{1} A_{2}}+x_{2} X_{B_{2}} \Delta^{A_{1} B_{2}}+x_{2} X_{C_{2}}+x_{2} X_{D_{2}} \Delta^{A_{1} D_{2}}\right)} \\
X_{A_{2}}=\frac{1}{1+\rho\left(x_{1} X_{A_{1}} \Delta^{A_{1} A_{2}}+x_{2} X_{C_{2}} \Delta^{A_{2} C_{2}}+x_{2} X_{D_{2}} \Delta^{A_{2} D_{2}}\right)} \\
X_{B_{2}}=\frac{1}{1+\rho\left(x_{1} X_{A_{1}} \Delta^{A_{1} A_{2}}+x_{2} X_{C_{2}} \Delta^{B_{2} C_{2}+x_{2} X_{D_{2}} \Delta^{B_{2} D_{2}}}\right)} \\
X_{C_{2}}=\frac{1}{1+\rho\left(x_{1} X_{A_{1}} \Delta^{A_{1} C_{2}}+x_{2} X_{A_{2}} \Delta^{A_{2} C_{2}}+x_{2} X_{B_{2}} \Delta^{B_{2} C_{2}}\right)}
\end{gathered}
$$




$$
X_{D_{2}}=\frac{1}{1+\rho\left(x_{1} X_{A_{1}} \Delta^{A_{1} D_{2}}+x_{2} X_{A_{2}} \Delta^{A_{2} D_{2}}+x_{2} X_{B_{2}} \Delta^{B_{2} D_{2}}\right)}
$$

\section{Mezclas de alcoholes}

Las mezclas de alcoholes son tratadas con los modelos de asociación $2 \mathrm{~B}$, exceptuando el metanol, ya que, como menciona Kontogeorgis y Folas (2010), este se representa con el esquema 3B. Las moléculas de alcoholes tienen un sitio aceptor y un sitio donador de protones tal como se muestra en la figura 3.

Acorde con las interacciones que se dan entre las moléculas de alcoholes, las fracciones $X_{\hat{A}_{j}}$ están dadas de la siguiente manera:

Sitio $A_{1}$ de una molécula i + Sitio $B_{1}$ de otra molécula i

Sitio $A_{1}$ de una molécula i + Sitio $B_{2}$ de una molécula j

Sitio $A_{2}$ de una molécula j + Sitio $B_{1}$ de una molécula i

Sitio $A_{2}$ de una molécula j + Sitio $B_{2}$ de otra molécula j

$$
\begin{aligned}
& X_{A_{1}}=\frac{1}{1+\rho\left(x_{1} X_{B_{1}} \Delta^{A_{1} B_{1}}+x_{2} X_{B_{2}} \Delta^{A_{1} B_{2}}\right)} \\
& X_{B_{1}}=\frac{1}{1+\rho\left(x_{1} X_{A_{1}} \Delta^{A_{1} B_{1}}+x_{2} X_{A_{2}} \Delta^{A_{2} B_{1}}\right)} \\
& X_{A_{2}}=\frac{1}{1+\rho\left(x_{1} X_{B_{1}} \Delta^{A_{2} B_{1}}+x_{2} X_{B_{2}} \Delta^{A_{2} B_{2}}\right)} \\
& X_{B_{2}}=\frac{1}{1+\rho\left(x_{1} X_{A_{1}} \Delta^{A_{1} B_{2}}+x_{2} X_{A_{2}} \Delta^{A_{2} B_{2}}\right)}
\end{aligned}
$$

\section{Mezclas de alcohol y agua}

Las mezclas entre alcoholes y agua, se representan por los esquemas de asociación $2 \mathrm{~B}$ y $4 \mathrm{C}$ respectivamente. Como se muestra en la figura 4, la molécula del alcohol consiste en un sitio aceptor y uno donador de protones, mientras la del agua presenta dos sitios aceptores y dos donadores de protones.

Para el cálculo de las fracciones $X_{A}$, las interacciones entre alcoholes y agua están dadas como: 
LÍNEA DE INVESTIGACIÓN: PROCESO DE SEPARACIÓN SIN REACCIÓN

Sitio $A_{1}$ de una molécula i + Sitio $B_{1}$ de otra molécula i

Sitio $A_{1}$ de una molécula i + Sitio $A_{2}$ de una molécula j

Sitio $A_{1}$ de una molécula i + Sitio $B_{2}$ de una molécula j

Sitio $A_{2}$ de una molécula j + Sitio $C_{2}$ de otra molécula j

Sitio $A_{2}$ de una molécula j + Sitio $D_{2}$ de otra molécula j

Sitio $B_{1}$ de una molécula $\mathrm{i}+$ Sitio $C_{2}$ de otra molécula j

Sitio $B_{1}$ de una molécula i + Sitio $D_{2}$ de una molécula j

Sitio $B_{2}$ de una molécula j + Sitio $C_{2}$ de otra molécula j

Sitio $B_{2}$ de una molécula j + Sitio $D_{2}$ de otra molécula j

Por lo tanto, las ecuaciones para el cálculo del término clave de asociación $X_{A_{j}}$ se expresan así:

$$
\begin{aligned}
& X_{A_{1}}=\frac{1}{1+\rho\left(x_{1} X_{B_{1}} \Delta^{A_{1} B_{1}}+x_{2} X_{A_{2}} \Delta^{A_{2} A_{2}}+x_{2} X_{B_{2}} \Delta^{A_{1} B_{2}}\right)} \\
& X_{B_{1}}=\frac{1}{1+\rho\left(x_{1} X_{A_{1}} \Delta^{A_{1} B_{1}}+x_{2} X_{C_{2}} \Delta^{B_{2} C_{2}}+x_{2} X_{D_{2}} \Delta^{B_{1} D_{2}}\right)} \\
& X_{A_{2}}=\frac{1}{1+\rho\left(x_{1} X_{A_{1}} \Delta^{A_{1} A_{2}}+x_{2} X_{C_{2}} \Delta^{A_{2} C_{2}}+x_{2} X_{D_{1}} \Delta^{A_{2} D_{2}}\right)} \\
& X_{A_{2}}=\frac{1}{1+\rho\left(x_{1} X_{A_{1}} \Delta^{A_{1} A_{2}}+x_{2} X_{C_{2}} \Delta^{A_{2} C_{2}}+x_{2} X_{D_{1}} \Delta^{A_{2} D_{2}}\right)} \\
& X_{C_{2}}=\frac{1}{1+\rho\left(x_{1} X_{B_{1}} \Delta^{B_{1} C_{2}}+x_{2} X_{A_{2}} \Delta^{A_{2} C_{2}}+x_{2} X_{B_{2}} \Delta^{B_{2} C_{2}}\right)} \\
& X_{D_{2}}=\frac{1}{1+\rho\left(x_{1} X_{B_{1}} \Delta^{B_{1} D_{2}}+x_{2} X_{A_{2}} \Delta^{A_{2} D_{2}}+x_{2} X_{B_{2}} \Delta^{B_{2} D_{2}}\right)}
\end{aligned}
$$

\section{CONCLUSIONES}

Se presentaron las ecuaciones para el cálculo del término de asociación en los modelos termodinámicos SAFT y CPA para los sistemas ácido + ácido, ácido + agua, alcohol + alcohol y alcohol + agua. Adicionalmente, se mostraron los esquemas de asociación correspondientes a cada tipo de 
sustancia que presentan los mejores resultados en el cálculo de propiedades de componente puro y en la predicción del equilibrio de fases.

\section{REFERENCIAS}

Chapman, W. G., Gubbins, K. E., Jackson, G., \& Radosz, M. (1989). SAFT: Equation-of-state solution model for associating fluids. Fluid Phase Equilibria, 52(C), 31-38. http://doi. org/10.1016/0378-3812(89)80308-5

Chapman, W. G., Gubbins, K. E., Jackson, G., \& Radosz, M. (1990). New reference equation of state for associating liquids. Industrial \& Engineering Chemistry Research, 29(8), 1709-1721. http://doi.org/10.1021/ie00104a021

Folas, G. K. (2006). Modeling of Complex Mixtures Containing Hydrogen Bonding Molecules (Doctoral thesis, Technical University of Denmark). Tomado de http://orbit.dtu.dk/en/publications/ modeling-of-complex-mixtures-containing-hydrogen-bonding-molecules(fbf80685-fae84efa-974f-ea7202e5c856).html

Huang, S. H., \& Radosz, M. (1990). Equation of state for small, large, polydisperse and associating molecules. Ind. Eng. Chem. Res., 29, 2284-2294. http://doi.org/10.1021/ie00107a014

Kontogeorgis, G. M., Voutsas, E. C., Yakoumis, I. V., \& Tassios, D. P. (1996). An Equation of State for Associating Fluids. Industrial \& Engineering Chemistry Research, 35(11), 4310-4318. http:// doi.org/10.1021/ie9600203

Kontogerogis, G. M., \& Folas, G. K. (2010). Thermodynamic models for industrial applications - from classical and advanced mixing rules to association theories. United Kingdom: John Wiley \& Sons Ltd.

Michelsen, M. L., \& Hendriks, E. M. (2001). Physical properties from association models. Fluid Phase Equilibria, 180(1-2), 165-174. http://doi.org/10.1016/S0378-3812(01)00344-2

Michelsen, M. L. (2006). Robust and efficient solution procedures for association models. Industrial and Engineering Chemistry Research, 45(25), 8449-8453. http://doi.org/10.1021/ie060029x 
LÍNEA DE INVESTIGACIÓN: PROCESO DE SEPARACIÓN SIN REACCIÓN

\section{ANEXO}

Tabla 1

Esquemas de asociación de Huang y Radosz

\begin{tabular}{|c|c|c|c|}
\hline Especies & Formula & Tipo Riguroso & Tipo Asignado \\
\hline Ácido & - & $1 \mathrm{~A}$ & $1 \mathrm{~A}$ \\
\hline Alcohol & $-\ddot{O H}_{\mathrm{CH}}^{\mathrm{A}}$ & 3B & $2 \mathrm{~B}$ \\
\hline Agua & $B: \begin{array}{l}A \\
\ddot{H}: H C\end{array}$ & $4 \mathrm{C}$ & 3B \\
\hline \multicolumn{4}{|c|}{ Aminas } \\
\hline Ternarias & $-\ddot{N}-$ & $1 \mathrm{~A}$ & no auto asociante \\
\hline Secundarias & $-\underset{H}{H} \frac{A}{B}$ & $2 \mathrm{~B}$ & $2 \mathrm{~B}$ \\
\hline Primarias & $\begin{array}{c}\ddot{C} \\
-\ddot{N}: H B \\
\ddot{H} A\end{array}$ & 3B & 3B \\
\hline Amoniaco & 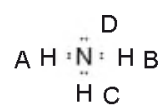 & $4 \mathrm{~B}$ & 3B \\
\hline
\end{tabular}

Fuente: Huang y Radosz, 1990.

Tabla 2

Fracciones $X_{A}$ para diferentes tipos de sitios

\begin{tabular}{llll}
\hline Tipo & Aproximación $\Delta$ & Aproximación $X_{A}$ & $X_{A}$ \\
\hline $1 A$ & $\Delta^{A A} \neq 0$ & & $\frac{-1+(1+4 \rho \Delta)^{\frac{1}{2}}}{2 \rho \Delta}$ \\
$2 \mathrm{~A}$ & $\Delta^{A A}=\Delta^{A B}=\Delta^{B B} \neq 0$ & $X_{A}=X_{B}$ & $\frac{-1+(1+8 \rho \Delta)^{\frac{1}{2}}}{4 \rho \Delta}$ \\
$2 B$ & $\Delta^{A A}=\Delta^{B B}=0$ & $X_{A}=X_{B}$ & $-1+(1+8 \rho \Delta)^{\frac{1}{2}}$ \\
& $\Delta^{A B} \neq=0$ & & $2 \rho \Delta$ \\
$3 A$ & $\Delta^{A A}=\Delta^{A B}=\Delta^{B B}=\Delta^{A C}=\Delta^{B C}$ & $X_{A}=X_{B}=X_{C}$ & $-1+(1+12 \rho \Delta)^{\frac{1}{2}}$ \\
& $=\Delta^{C C} \neq 0$ & & $6 \rho \Delta$ \\
\hline
\end{tabular}




\begin{tabular}{|c|c|c|c|}
\hline Tipo & Aproximación $\Delta$ & Aproximación $X_{A}$ & $X_{A}$ \\
\hline \multirow{2}{*}{$3 \mathrm{~B}$} & $\Delta^{A A}=\Delta^{A B}=\Delta^{B B}=\Delta^{C C}=0$ & $X_{A}=X_{B}$ & $-1(1-\rho \Delta)+\left((1+\rho \Delta)^{2}+4 \rho \Delta\right)^{\frac{1}{2}}$ \\
\hline & $\Delta^{A C}=\Delta^{B C} \neq 0$ & $X_{C}=2 X_{A}-1$ & $\bar{P}$ \\
\hline $4 \mathrm{~A}$ & $\begin{array}{l}\Delta^{A A}=\Delta^{A B}=\Delta^{B B}=\Delta^{A C}=\Delta^{B C} \\
=\Delta^{C C}=\Delta^{A D}=\Delta^{B D} \\
=\Delta^{C D}=\Delta^{D D} \neq 0\end{array}$ & $X_{A}=X_{B}=X_{C}=X_{D}$ & $\frac{-1+(1+16 \rho \Delta)^{\frac{1}{2}}}{8 \rho \Delta}$ \\
\hline \multirow[t]{2}{*}{$4 \mathrm{~B}$} & $\begin{array}{l}\Delta^{A A}=\Delta^{A B}=\Delta^{B B}=\Delta^{A C}=\Delta^{B C} \\
=\Delta^{C C}=\Delta^{D D}=0\end{array}$ & $X_{A}=X_{B}=X_{C}$ & $-(1-2 \rho \Delta)+\left((1+2 \rho \Delta)^{2}+4 \rho \Delta\right)$ \\
\hline & $\Delta^{A D}=\Delta^{B D}=\Delta^{C D} \neq 0$ & $X_{D}=3 X_{A}-2$ & $6 \rho \Delta$ \\
\hline $4 C$ & $\begin{array}{l}\Delta^{A A}=\Delta^{A B}=\Delta^{B B}=\Delta^{C C}=\Delta^{C D} \\
=\Delta^{D D}=0 \\
\Delta^{A C}=\Delta^{A D}=\Delta^{B C}=\Delta^{B D} \neq 0\end{array}$ & $X_{A}=X_{B}=X_{C}=X_{D}$ & $\frac{-1+(1+8 \rho \Delta)^{\frac{1}{2}}}{4 \rho \Delta}$ \\
\hline
\end{tabular}

Fuente: Huang y Radosz, 1990

Tabla 3

Mezclas de esquemas de asociación

\begin{tabular}{|c|c|c|c|}
\hline Mezcla & Componente 1 & Componente 2 & Valores de $\Delta$ \\
\hline $1 A+1 A$ & & & $\begin{array}{l}\Delta^{A_{1} A_{1}}, \Delta^{A_{1} A_{2}}, \Delta^{A_{2} A_{2}} \neq 0 \\
\text { Otros }=0\end{array}$ \\
\hline $1 A+4 C$ & & $A_{2}$ & $\begin{array}{l}\Delta^{A_{1} A_{1}}, \Delta^{A_{1} A_{2}}, \Delta^{A_{1} B_{2}}, \Delta^{A_{1} C_{2}}, \Delta^{A_{1} D_{2}}, \Delta^{A_{2} C_{2}}, \Delta^{A_{2} D_{2}}, \Delta^{B_{2} C_{2}}, \Delta^{B_{2} D_{2}} \neq 0 \\
\text { Otros }=0\end{array}$ \\
\hline $2 B+2 B$ & $B_{1}$ & $\mathrm{R}-$ & $\begin{array}{l}\Delta^{A_{1} B_{1}}, \Delta^{A_{1} B_{2}}, \Delta^{A_{2} B_{1}}, \Delta^{A_{2} B_{2}} \neq 0 \\
\text { Otros }=0\end{array}$ \\
\hline $2 B+4 C$ & $B^{H}$ & $\mathrm{O}_{\mathrm{H}_{2}}^{\mathrm{C}_{2}}:_{\mathrm{B}_{2}}^{\mathrm{D}_{2}}$ & $\begin{array}{l}\Delta^{A_{1} B_{1}}, \Delta^{A_{1} A_{2}}, \Delta^{A_{1} B_{2}}, \Delta^{B_{1} C_{2}}, \Delta^{B_{1} D_{2}}, \Delta^{A_{2} C_{2}}, \Delta^{A_{2} D_{2}}, \Delta^{B_{2} C_{2}}, \Delta^{B_{2} D_{2}} \neq 0 \\
\text { Otros }=0\end{array}$ \\
\hline
\end{tabular}

Fuente: Los autores 
LIINEA DE INVESTIGACIÓN: PROCESO DE SEPARACIÓN SIN REACCIÓN

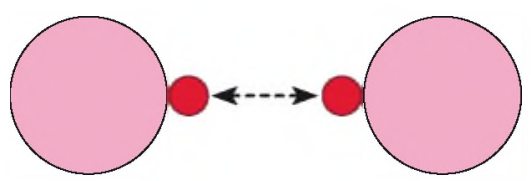

Figura 1. Asociación entre ácidos

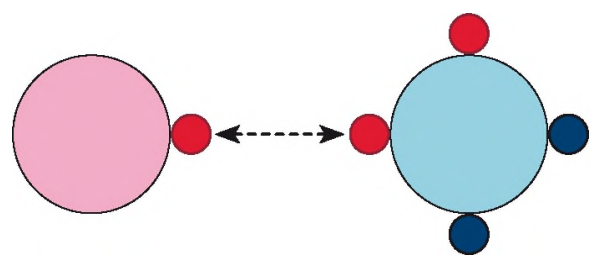

Sitio Aceptor de Protones

Sitio Donador de Protones

Figura 2. Asociación entre ácido y agua

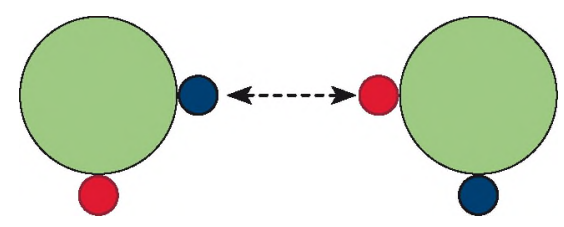

Sitio Aceptor de Protones

Sitio Donador de Protones

Figura 3. Asociación entre alcoholes

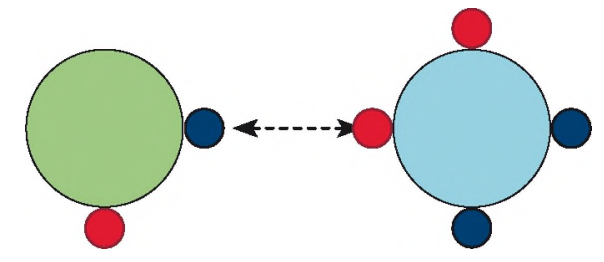

Sitio Aceptor de Protones

Sitio Donador de Protones

Figura 4. Asociación entre alcohol y agua

Fuente: Los autores 